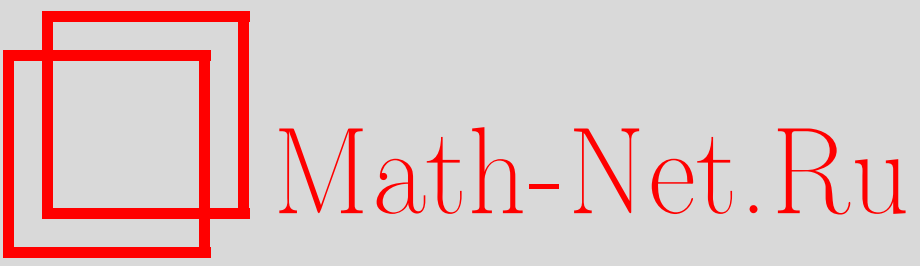

Н. Г. Иноземцева, Б. И. Садовников, Нелокальные гидродинамические уравнения и преобразование ББГКИ-иерархии для твердых сфер, ТМФ, 1999, том 120, номер 3, 394-399

DOI: https://doi.org/10.4213/tmf786

Использование Общероссийского математического портала Math-Net.Ru подразумевает, что вы прочитали и согласны с пользовательским соглашением

http: //www . mathnet.ru/rus/agreement

Параметры загрузки:

IP : 54.210 .77 .194

26 апреля 2023 г., 07:03:15 


\author{
ТЕОРЕТИЧЕСКАЯ \\ И МАТЕМАТИЧЕСКАЯ \\ ФИЗИКА \\ Том 120, № 3 \\ сентябрь, 1999
}

(C) 1999 г

Н.Г. Иноземцева*, Б. И. Садовников*

\title{
НЕЛОКАЛЬНЫЕ ГИДРОДИНАМИЧЕСКИЕ УРАВНЕНИЯ И ПРЕОБРАЗОВАНИЕ ББГКИ-ИЕРАРХИИ ДЛЯ ТВЕРДЫХ СФЕР
}

На основе бесконечной цепочки уравнений для функций распределения в случае ББГКИ-иерархии исследуется вопрос получения кинетического уравнения для классических систем твердых сфер. Хорошо известно, что гипотеза о полной синхронизации всех распределений ведет к некоторым трудностям при описании "хвостов" автокорреляционных функций и других корреляционных эффектов при средних и высоких плотностях. Показано, как можно избежать этих трудностей с помощью сохранения явного вида зависящих от времени динамических корреляций в схеме ББГКИ-замыкания.

Проблема точного математического решения кинетического уравнения Больцмана с самого начала века притягивала к себе умы лучших физиков и математиков. Н. Н. Боголюбов был одним из первых, кто внес реальный вклад в ее решение. В своей монографии [1] он предложил в качестве основополагающей идеи рассматривать динамическую многочастичную задачу с точки зрения бесконечной системы редуцированных функций распределения (названной впоследствии ББГКИ-иерархией), что эквивалентно в термодинамическом пределе уравнению Лиувилля. Более того, он предложил регулярную схему замыкания этой бесконечной системы, используя идею об ослабленных многочастичных корреляциях и синхронизации всех высших функций распределения с первой. Данная схема аналогична разложению по малому параметру $\alpha=n a^{3}$, где $n$-плотность и $a$ - характерный размер области эффективного двухчастичного взаимодействия.

Будучи достаточно ясной с физической точки зрения, эта схема позволяет получить классическое уравнение Больцмана в низшем порядке по $\alpha$ и поставить вопрос о вычислении очень сложных поправок более высокого порядка. Главная сложность при этом состоит в необходимости трактовки поведения $(l+1)$-частичных комплексов для получения решения в 1 -м порядке.

Система твердых сфер особенно удобна для этих целей как наиболее простая с точки зрения двухчастичной динамики [2]. Во втором порядке по $\alpha$ соответствующие вычисления были проведены Чо и Уленбеком [3]. Много позже, однако, при изучении поправок третьего порядка было обнаружено, что некоторые члены в них являются расходящимися [4]. Использование методов численного моделирования позволило установить, что скоростные автокорреляционные функции в достаточно плотных системах затухают не-

\footnotetext{
* Московский государственный университет, Москва, Россия
} 
ожиданно медленно [5]. Все это подтверждает гипотезу о важной роли дальнодействующих динамических корреляций, которые не принимались в расчет при построении оригинальной первоначальной теории Боголюбова. Позднее данное допушение было подтверждено при исследовании свойств достаточно плотных систем твердых сфер и дисков в гидродинамическом режиме.

В середине восьмидесятых Боголюбов возвратился к этой старой проблеме и сформулировал новую, достаточно парадоксальную схему приближенного замыкания ББГКИ-иерархии для твердых сфер. Точкой отсчета при этом была бесконечная ББГКИ-система уравнений

$$
\begin{aligned}
\left(\frac{\partial}{\partial t}+L(1)\right) f_{1}\left(t, x_{1}\right) & =n \int d x_{2} \widetilde{T}(1,2) f_{2}\left(t, x_{1}, x_{2}\right) \\
\left(\frac{\partial}{\partial t}+L(1,2)\right) f_{2}\left(t, x_{1}, x_{2}\right) & =n \int d x_{3}[\widetilde{T}(1,3)+\widetilde{T}(2,3)] f_{3}\left(t, x_{1}, x_{2}, x_{3}\right),
\end{aligned}
$$

где

$$
x_{j}=\left(\vec{r}_{j}, \vec{v}_{j}\right), \quad L(j)=\vec{v}_{j} \frac{\partial}{\partial \vec{r}_{j}}, \quad L(1,2)=L(1)+L(2)-\widetilde{T}(1,2)
$$

и $\widetilde{T}(i, j)$ - оператор парных столкновений,

$$
\widetilde{T}(i, j)=a^{2} \int_{\left(\vec{v}_{i}-\vec{v}_{j}\right) \vec{\sigma}>0} d \sigma\left(\vec{v}_{i}-\vec{v}_{j}\right) \vec{\sigma}\left[\delta\left(\vec{r}_{i}-\vec{r}_{j}-a \vec{\sigma}\right) b_{\vec{\sigma}}^{(i, j)}-\delta\left(\vec{r}_{i}-\vec{r}_{j}+a \vec{\sigma}\right)\right] .
$$

Оператор $b$ действует на скорости, изменяя их в процессе парных столкновений,

$$
\vec{v}_{i}^{*}=\vec{v}_{i}-\vec{\sigma}\left(\vec{v}_{i}-\vec{v}_{j}\right) \vec{\sigma}, \quad \vec{v}_{j}^{*}=\vec{v}_{j}+\vec{\sigma}\left(\vec{v}_{i}-\vec{v}_{j}\right) \vec{\sigma} .
$$

Кластерное разложение ББГКИ-схемы линеаризует условие ослабления корреляций,

$$
\begin{aligned}
f_{2}(t, 1,2) & =f_{1}(t, 1) f_{1}(t, 2)+g_{2}(t, 1,2), \\
f_{3}(t, 1,2,3) & =f_{1}(t, 1) f_{2}(t, 2) f_{3}(t, 3)+\sum_{i=1 ; j>k ; k \neq i}^{3} f_{1}(t, i) g_{2}(t, j, k)+g_{3}(t, 1,2,3),
\end{aligned}
$$

но при этом делает систему нелинейной. Боголюбов [6] отметил, что дальние динамические корреляции появляются, если пренебречь слагаемым с $g_{3}$ в соответствующих уравнениях. В этом случае получается замкнутая, но нелинейная система двух уравнений для $f_{1}$ и $g_{2}$. Более того, оказывается, что в низшем порядке по $\alpha$ можно опустить оператор столкновений в левой части уравнения (2). В результате система приобретает вид

$$
\begin{gathered}
\left(\frac{\partial}{\partial t}+L(1)\right) f_{1}\left(t, x_{1}\right)=n \int d x_{2} \widetilde{T}(1,2)\left[f_{1}\left(t, x_{1}\right) f_{1}\left(t, x_{2}\right)+g_{2}\left(t, x_{1}, x_{2}\right)\right] \\
\left(\frac{\partial}{\partial t}+\mathscr{L}(t, 1)+\mathscr{L}(t, 2)\right) g_{2}\left(t, x_{1}, x_{2}\right)=\widetilde{T}(1,2) f_{1}\left(t, x_{1}\right) f_{1}\left(t, x_{2}\right)
\end{gathered}
$$

где

$$
\mathscr{L}(t, i) \psi\left(x_{i}\right)=L(i) \psi\left(x_{i}\right)=n \int d x_{3} \widetilde{T}(i, 3)\left[f_{1}\left(t, x_{i}\right) \psi\left(x_{3}\right)+f_{1}\left(t, x_{3}\right) \psi\left(x_{i}\right)\right]
$$


Можно заметить, что система (4), (5) сильно нелинейна. В приближении $g_{2}\left(t, x_{1}, x_{2}\right)=0$ мы получаем обычное уравнение Больцмана-Энскога. Позднее было показано, что система (4), (5) при первоначальном условии $g_{2}\left(t, x_{1}, x_{2}\right)=0$ после исключения $g_{2}$ из выражения (4) с помошью (5) должна рассматриваться как нелокальное кинетическое уравнение, содержашее $T$-упорядочение некоммутирующих, зависящих от времени операторов $[7]$,

$$
\begin{aligned}
g_{2}\left(t, x_{1}, x_{2}\right) & =\int_{0}^{t} Q(t) Q^{-1}(\tau) \widetilde{T}(1,2) f_{1}\left(\tau, x_{1}\right) f_{1}\left(\tau, x_{2}\right) d \tau, \\
Q(t) & =T \exp \left(\int_{0}^{t}(\mathscr{L}(\tau, 1)+\mathscr{L}(\tau, 2)) d \tau\right),
\end{aligned}
$$

где $T$ означает хронологическое упорядочение.

В обшем случае достаточно сложно анализировать свойства этого уравнения. Однако реально оказывается важным исследовать только роль дальних динамических корреляций. Для этой цели наличия информации о поведении решений в гидродинамическом режиме вполне достаточно.

Обычная схема построения гидродинамического приближения в системе (4), (5) ведет, как было показано в работе [8], к расходимости на стадии вычислений коэффициентов Барнета и, таким образом, результата не дает. Здесь мы выберем другой метод, основанный на использовании формального гомогенного параметра $\mu$, добавленного к слагаемым в $(4),(5)$, содержашим временные и пространственные производные,

$$
\begin{gathered}
\mu\left(\frac{\partial f_{1}(t, 1)}{\partial t}+\vec{v}_{1} \frac{\partial}{\partial \vec{r}_{1}} f_{1}(t, 1)\right)=n \int d x_{2} \widetilde{T}(1,2)\left[f_{1}(t, 1) f_{1}(t, 2)+g_{2}(t, 1,2)\right] \\
\mu\left(\frac{\partial g_{2}(t, 1,2)}{\partial t}+\frac{1}{2}\left(\vec{v}_{1}+\vec{v}_{2}\right) \frac{\partial}{\partial \vec{R}} g_{2}(t, 1,2)\right)+\left(\vec{v}_{1}-\vec{v}_{2}\right) \frac{\partial}{\partial \vec{\rho}} g_{2}(t, 1,2)= \\
=n \sum_{i=1}^{2} \int d x_{3} \widetilde{T}(i, 3)\left[f_{1}(t, i) g_{2}(t, 3-i, 3)+f_{1}(t, 3) g_{2}(t, 1,2)\right]+ \\
+T(1,2) f_{1}(t, 1) f_{1}(t, 2), \\
\vec{R}=\frac{1}{2}\left(\vec{r}_{1}+\vec{r}_{2}\right), \quad \vec{\rho}=\vec{r}_{1}-\vec{r}_{2} .
\end{gathered}
$$

Когда $\mu=0$, решение уравнений $(8),(9)$ дается выражением $f_{1}(1)=f_{\mathrm{M}}(1), g_{2}(1,2)=0$, где $f_{\mathrm{M}}(1)$ - равновесная функция распределения. Следовательно, можно получить приближенное решение при $\mu \neq 0, \mu \ll 1$ в виде

$$
\begin{aligned}
f_{1}(t, 1) & =f_{\mathrm{M}}(t, 1)\left(1+\mu \phi_{1}(t, 1 \mid \mu)\right), \\
g_{2}(t, 1,2) & =f_{\mathrm{M}}\left(t, \vec{\xi}_{1}, \vec{v}_{1}\right) f_{\mathrm{M}}\left(t, \vec{\xi}_{2}, \vec{v}_{2}\right) \mu \psi_{1}(t, 1,2 \mid \mu),
\end{aligned}
$$

где $\vec{\xi}_{1,2}=\vec{R} \pm \mu \frac{\vec{\rho}}{2}$ и

$$
f_{\mathrm{M}}(t, 1)=\rho\left(\vec{r}_{1}, t\right)\left(\frac{m}{2 \pi \theta\left(\vec{r}_{1}, t\right)}\right)^{3 / 2} \exp \left\{-\frac{m\left(\vec{v}_{1}-\vec{u}\left(\vec{r}_{1}, t\right)\right)^{2}}{2 \theta\left(r_{1}, t\right)}\right),
$$

а $\rho, \theta, \vec{u}$-соответственно плотность, температура и макроскопическая скорость вблизи положения равновесия. 
Эти функции, как это следует из (4), (5), подчиняются следующим “законам сохранения":

где

$$
\begin{aligned}
\frac{\partial \rho}{\partial t}+\frac{\partial}{\partial \vec{r}}(\rho \vec{u}) & =0 \\
\rho\left(\frac{\partial}{\partial t}+u_{k} \frac{\partial}{\partial r_{k}}\right) u_{j} & =-\frac{\partial p_{i k}}{\partial r_{k}} \\
\frac{3}{2} \rho\left(\frac{\partial}{\partial t}+u_{k} \frac{\partial}{\partial r_{k}}\right) \theta & =-\left(p_{i k}\left(\frac{\partial u_{i}}{\partial r_{k}}+\frac{\partial u_{k}}{\partial r_{i}}\right)+\frac{\partial q_{k}}{\partial r_{k}}\right),
\end{aligned}
$$

$$
\begin{aligned}
p_{i k} & =\int\left(v_{i}-u_{i}\right)\left(v_{k}-u_{k}\right) f_{1}(t, 1) d \vec{v}_{1}, \\
q_{i} & =\frac{1}{2} \int\left(v_{i}-u_{i}\right)\left(v_{k}-u_{k}\right)^{2} f_{1}(t, 1) d \vec{v}_{1} .
\end{aligned}
$$

Проблема построения гидродинамических уравнений состоит в нахож дении приближенного решения уравнений (8), (9) в форме (10) и в вычислении интегралов, стояших в правой части выражений (13). После подстановки (10) в (8), учитывая соотношение $\widetilde{T}(i, j) f_{\mathrm{M}}(t, i) f_{\mathrm{M}}(t, j)=f_{\mathrm{M}}(t, i) f_{\mathrm{M}}(t, j) \widetilde{T}(i, j)$, получаем

$$
\begin{aligned}
& {\left[f_{\mathrm{M}}(t, 1)\right]^{-1}\left[\frac{\partial}{\partial t}+\vec{v}_{1} \frac{\partial}{\partial \vec{r}_{1}}\right] f_{\mathrm{M}}(t, 1)=} \\
& \quad=n \Lambda\left(1, \vec{r}_{1}\right) \phi_{1}(t, 1)+n \int d \vec{v}_{2} f_{\mathrm{M}}\left(\vec{r}_{1}, \vec{v}_{2}\right) T(1,2) \psi_{1}\left(t, \vec{r}_{1}, 0, \vec{v}_{1}, \vec{v}_{2}\right)
\end{aligned}
$$

где введены обозначения

$$
\begin{aligned}
& \psi_{1}\left(t, \vec{R}, \vec{\rho}, \vec{v}_{1}, \vec{v}_{2}\right)=\psi_{1}(t, 1,2 \mid \mu), \quad \widetilde{T}(i, j)=T(i, j) \delta\left(\vec{r}_{1}-\vec{r}_{2}\right), \\
& \Lambda(i, \vec{\xi}) Y\left(\vec{v}_{i}\right)=\int d \vec{v}_{3} f_{\mathrm{M}}\left(\vec{\xi}, \vec{v}_{3}\right) T(i, 3)\left[Y\left(\vec{v}_{i}\right)+Y\left(\vec{v}_{3}\right)\right], \quad i=1,2 .
\end{aligned}
$$

Заметим, что уравнение (14) не содержит явной зависимости от параметра $\mu$. Второе уравнение соответствуюшей системы приобретает вид

$$
\begin{aligned}
{\left[\mu \left(\frac{\partial}{\partial t}+\right.\right.} & \left.\frac{1}{2}\left(\vec{v}_{1}+\vec{v}_{2}\right) \frac{\partial}{\partial \vec{R}}\right)+\left(\vec{v}_{1}-\vec{v}_{2}\right) \frac{\partial}{\partial \vec{\rho}}+\mu L\left(t, \vec{R}, \vec{\rho}, \vec{v}_{1}, \vec{v}_{2}\right)- \\
& \left.-n\left(\Lambda\left(1, \vec{\xi}_{1}\right)+\Lambda\left(2, \vec{\xi}_{2}\right)\right)\right] \psi_{1}\left(t, \vec{R}, \vec{\rho}, \vec{v}_{1}, \vec{v}_{2}\right)= \\
= & \delta(\vec{\rho}) T(1,2)\left[\phi_{1}\left(t, \vec{R}, \vec{v}_{1}\right)+\phi_{1}\left(t, \vec{R}, \vec{v}_{2}\right)\right] .
\end{aligned}
$$

Здесь мы использовали обозначение

$$
L\left(t, \vec{R}, \vec{\rho}, \vec{v}_{1}, \vec{v}_{2}\right)=\left[\frac{\partial}{\partial t}+\frac{1}{2}\left(\vec{v}_{1}+\vec{v}_{2}\right) \frac{\partial}{\partial \vec{R}}+\mu^{-1}\left(\vec{v}_{1}-\vec{v}_{2}\right) \frac{\partial}{\partial \vec{\rho}}\right] \ln f_{\mathrm{M}}\left(\vec{\xi}_{1}, \vec{v}_{1}\right) f_{\mathrm{M}}\left(\vec{\xi}_{2}, \vec{v}_{2}\right) .
$$

Оставляя в уравнении (15) слагаемые нулевого и первого порядков по $\mu$, находим

$$
\begin{aligned}
&\left\{\left(\vec{v}_{1}-\right.\right.\left.\vec{v}_{2}\right) \frac{\partial}{\partial \vec{\rho}}-n(\Lambda(1, \vec{R})+\Lambda(2, \vec{R}))+\mu\left[\frac{\partial}{\partial t}+\frac{1}{2}\left(\vec{v}_{1}+\vec{v}_{2}\right) \frac{\partial}{\partial \vec{R}}-n \frac{\vec{\rho}}{2} \times\right. \\
&\left.\left.\quad \times\left(\frac{\partial \Lambda(1, \vec{R})}{\partial \vec{R}}-\frac{\partial \Lambda(2, \vec{R})}{\partial \vec{R}}\right)+L_{0}\right]\right\} \psi_{1}\left(t, \vec{R}, \rho, \vec{v}_{1}, \vec{v}_{2}\right)= \\
&=\delta(\rho) T(1,2)\left[\phi_{1}\left(t, \vec{R}, \vec{v}_{1}\right)+\phi_{1}\left(t, \vec{R}, \vec{v}_{2}\right)\right]
\end{aligned}
$$


где

$$
\begin{aligned}
L_{0} & =\sum_{i=1}^{2}\left[f_{\mathrm{M}}\left(\vec{R}, \vec{v}_{i}\right)\right]^{-1}\left[\frac{\partial}{\partial t}+\vec{v}_{i} \frac{\partial}{\partial \vec{R}}\right] f_{\mathrm{M}}\left(\vec{R}, \vec{v}_{i}\right), \\
\frac{\partial \Lambda(1, \vec{R})}{\partial \vec{R}} Y\left(\vec{v}_{1}\right) & =\int d \vec{v}_{3} \frac{\partial f_{\mathrm{M}}\left(\vec{R}, \vec{v}_{3}\right)}{\partial \vec{R}} T(1,3)\left[Y\left(\vec{v}_{1}\right)+Y\left(\vec{v}_{3}\right)\right] .
\end{aligned}
$$

Выражая в (14), (16) слагаемые $\partial f_{\mathrm{M}} / \partial t$ с помошью гидродинамических уравнений нулевого порядка (что соответствует $p_{i k}=m^{-1} \theta \delta_{i k}, \vec{q}=0$ в (12)), получаем

$$
\begin{aligned}
n \Lambda(1, \vec{r}) & \phi_{1}\left(t, \vec{r}, \vec{v}_{1}\right)+n \int d \vec{v}_{2} f_{\mathrm{M}}\left(\vec{r}, \vec{v}_{2}\right) T(1,2) \psi_{1}\left(t, \vec{r}, 0, \vec{v}_{1}, \vec{v}_{2}\right)= \\
= & (2 \theta)^{-1} \frac{\partial \theta}{\partial r_{k}} A_{k}\left(\vec{V}_{1}\right)+\frac{m}{\theta} S_{i k} B_{i k}\left(\vec{V}_{1}\right), \\
{\left[\mu \left(\frac{\partial}{\partial t}+\right.\right.} & \left.\frac{1}{2}\left(\vec{v}_{1}+\vec{v}_{2}\right) \frac{\partial}{\partial \vec{R}}-n \frac{\vec{\rho}}{2}\left(\frac{\partial \Lambda(1, \vec{R})}{\partial \vec{R}}-\frac{\partial \Lambda(2, \vec{R})}{\partial \vec{R}}\right)+L_{0}\right)+ \\
& \left.+\left(\vec{v}_{1}-\vec{v}_{2}\right) \frac{\partial}{\partial \vec{\rho}}-n(\Lambda(1, \vec{R})+\Lambda(2, \vec{R}))\right] \psi_{1}\left(t, \vec{R}, \vec{\rho}, \vec{v}_{1}, \vec{v}_{2}\right)= \\
= & \delta(\vec{\rho}) T(1,2)\left[\phi_{1}\left(t, \vec{R}, \vec{v}_{1}\right)+\phi_{1}\left(t, \vec{R}, \vec{v}_{2}\right)\right],
\end{aligned}
$$

где

$$
\begin{aligned}
L_{0} & =(2 \theta)^{-1} \frac{\partial \theta}{\partial r_{k}} \sum_{\alpha=1}^{2} A_{k}\left(\vec{V}_{\alpha}\right)+\frac{m}{\theta} S_{i k} \sum_{\alpha=1}^{2} B_{i k}\left(\vec{V}_{\alpha}\right), \\
A_{k}\left(\vec{V}_{\alpha}\right) & =V_{k \alpha}\left(m \theta^{-1} \vec{V}_{\alpha}^{2}-5\right), \quad B_{i k}\left(\vec{V}_{\alpha}\right)=V_{i \alpha} V_{k \alpha}-\frac{1}{3} \delta_{i k} \vec{V}_{\alpha}^{2}, \\
V_{\alpha} & =v_{\alpha}-u_{\alpha}(\vec{r}, t), \quad S_{i k}=\frac{\partial u_{i}}{\partial r_{k}}+\frac{\partial u_{k}}{\partial r_{i}} .
\end{aligned}
$$

Заметим, что система (17), (18) линейна. Если пренебречь слагаемым, линейным по $\mu$ в уравнении (18), то его решение будет содержать расходимости [8]. Оставляя это слагаемое, решение уравнения (18) можно записать в виде

$$
\psi_{1}\left(t, \vec{R}, \vec{\rho}, \vec{v}_{1}, \vec{v}_{2}\right)=\mu^{-1} \int_{0}^{t} \widehat{Q}(t, \mu) \widehat{Q}^{-1}(\tau, \mu) \delta(\vec{\rho}) T(1,2)\left[\phi_{1}\left(t, \vec{R}, \vec{v}_{1}\right)+\phi_{1}\left(t, \vec{R}, \vec{v}_{2}\right)\right] d \tau
$$

где

$$
\begin{aligned}
\widehat{Q}(t, \mu)= & T \exp \int_{0}^{t}\left\{-\frac{1}{2}\left(\vec{v}_{1}+\vec{v}_{2}\right) \frac{\partial}{\partial \vec{R}}-L_{0}-n \frac{\vec{\rho}}{2}\left(\frac{\partial \Lambda(1, \vec{R})}{\partial \vec{R}}-\frac{\partial \Lambda(2, \vec{R})}{\partial \vec{R}}\right)+\right. \\
& \left.+\mu^{-1}\left(\vec{v}_{1}-\vec{v}_{2}\right) \frac{\partial}{\partial \vec{\rho}}-n(\Lambda(1, \vec{R})+\Lambda(2, \vec{R}))\right\} d \tau .
\end{aligned}
$$

Отметим, что все операторы и слагаемое $L_{0}$ в выражении (19) должны быть взяты при $t=\tau$.

Теперь нетрудно получить решение уравнения (17):

$$
\phi_{1}\left(t, \vec{r}, \vec{v}_{1}\right)=\frac{1}{n\left(\Lambda_{0}(1, \vec{r})+\widehat{Z}\right)}\left[(2 \theta)^{-1} \frac{\partial \theta}{\partial r_{k}} A_{k}\left(\vec{V}_{1}\right)+\frac{m}{\theta} S_{i k} B_{i k}\left(\vec{V}_{1}\right)\right],
$$


где

$$
\begin{aligned}
\widehat{Z} Y\left(t, \vec{r}, \vec{v}_{1}\right)= & \mu^{-1} \int d \vec{v}_{2} f_{\mathrm{M}}\left(\vec{r}, \vec{v}_{2}\right) T(1,2) \int_{0}^{t} \widehat{Q}(t, \mu) \widehat{Q}^{-1}(\tau, \mu) \times \\
& \times\left.\delta(\vec{\rho}) T(1,2)\left[Y\left(\tau, \vec{r}, \vec{v}_{1}\right)+Y\left(\tau, \vec{r}, \vec{v}_{2}\right)\right] d \tau\right|_{\vec{\rho}=0} .
\end{aligned}
$$

На последней стадии решения необходимо выбрать главное слагаемое по $\mu$ при $\mu \rightarrow 0$, отбросить остальные слагаемые более низких порядков и положить $\mu=1$. Данная процедура требует нахождения всех интегралов в выражениях (19), (20), включая хронологически упорядоченные интегралы, которые тяжело поддаются вычислению. Тем не менее в простейших случаях типа линеаризованных гидродинамических уравнений данный метод позволяет получить много полезных результатов, таких как неаналитическая зависимость гидродинамических частот от волнового вектора и т.д. (см., например, paботу [8]).

В заключение хотелось бы еше раз подчеркнуть, что оригинальная гипотеза Боголюбова [1], подтверждая пригодность кинетического уравнения Больцмана в приближении малой плотности, не описывает дальние динамические корреляции, вызванные сильно скоррелированными многочастичными взаимодействиями. Это оказывается возможным сделать, лишь используя более сложный в техническом отношении способ редуцирования ББГКИ-иерархии, предложенный Боголюбовым много позже и примененный в данной работе для построения нелокальных гидродинамических уравнений, которые не содержат специфических расходимостей.

\section{Список литературы}

[1] Н. Н. Боголюбов. Проблемы динамической теории в статистической физике. М.: Гостехиздат, 1946.

[2] M. H. Ernst et al. Physica. 1969. V. 45. P. 127.

[3] C. Чо, Дж. Уленбек. Кинетическая теория явлений в плотных газах. Доп-е в кн.: Дж. Уленбек, Дж. Форд. Лекции по статистической механике. М.: Мир, 1965. С. 189-280.

[4] J.R. Dorfman, E. G.D. Cohen. Phys. Lett. 1965. V. 16. P. 124; J. Math. Phys. 1967. V. 8. P. 282.

[5] B. J. Alder, T. E. Wainwright. Phys. Rev. Lett. 1967. V. 18. P. 988.

[6] H. H. Боголюбов. Кинетические уравнения и функции Грина в статистической механике. В сб.: Международный симпозиум по избранным проблемам в статистической механике. JINR D17-11490. Дубна: ОИЯИ, 1978. С. 7.

[7] Н. Г. Иноземщева, Б. И. Садовников. Обобщенная гидродинамика и процессы переноса. В сб.: III Международный симпозиум по избранным проблемам в статистической механике. T. 1. JINR D17-84-850. Дубна: ОИЯИ, 1985. С. 310.

[8] Н. Г. Иноземцева, Б. И. Садовников. ТМФ. 1982. Т. 51. С. 142.

Поступила в редакцию 25.III.1999 г. 